\title{
Properties of contactless and contacted charging in MEMS capacitive switches
}

\author{
M. Koutsoureli ${ }^{\text {a,* }}$, L. Michalas ${ }^{\text {a }}$, P. Martins ${ }^{\mathrm{b}}$, E. Papandreou $^{\mathrm{a}}$, A. Leuliet $^{\mathrm{b}}$, S. Bansropun $^{\mathrm{b}}$, \\ G. Papaioannou ${ }^{\text {a }}$, A. Ziaei ${ }^{\text {b }}$ \\ a Solid State Section, Physics Department, University of Athens, Athens 15784, Greece \\ ${ }^{\mathrm{b}}$ Thales Research \& Technology, F91767 Palaiseau Cedex, France
}

\section{A R T I C L E I N F O}

\section{Article history:}

Received 24 May 2013

Accepted 9 July 2013

\begin{abstract}
A B S T R A C T
The dielectric charging in MEMS capacitive switches is a complex effect. The high electric field during pull-down causes intrinsic free charge migration and dipole orientation as well as charge injection. The macroscopic dipole moment of the first two mechanisms is opposite to the one arising from charge injection. This causes partial compensation hence mitigates the overall charging and increases the device lifetime. The charging due to intrinsic free charge migration and dipole orientation can be monitored under contactless electric field application in the pull-up state. The paper investigates the characteristics of contactless charging and compares them with the ones of contacted charging. The characteristics of the discharging process that follows each charging procedure are also presented.
\end{abstract}

(c) 2013 Elsevier Ltd. All rights reserved.

\section{Introduction}

Micro-electro-mechanical-systems (MEMS) technology has already emerged as an enabling technology for a new generation of high-performance RF components such as RF MEMS switches, tunable capacitors and inductors. In addition, RF MEMS components can be fully integrated with monolithic microwave integrated circuits and therefore can potentially lead to systems with small size, smaller weight, low power consumption and mass production $[1,2]$. Among the mentioned RF MEMS devices, the MEMS capacitive switch is a key device due to its unique RF performance compared to the current existing devices. The unique characteristics of MEMS capacitive switches make them ideal candidates for integration into passive circuits, such as phase shifters or tunable filters, for implementation in many terrestrial and space applications, including portable telecommunication, wireless computer networks, reconfigurable antennas and others.

However, the commercialization of the electrostatic MEMS capacitive switch is still hindered by reliability issues, especially the dielectric charging phenomenon [3]. The dielectric charging has been principally monitored through the number of switching cycles for different operating bias [4] and ambient conditions [5-7] and dielectric materials and substrates, a detailed presentation of all these being given in $[8,9]$.

\footnotetext{
* Corresponding author. Tel.: +30 210727 6722; fax: +30 2107276711 .

E-mail address: mkoutsoureli@phys.uoa.gr (M. Koutsoureli).
}

Regarding the dielectric charging mechanisms two mechanisms have been identified up to now, the contacted charging, where charges are injected under the presence of high electric field during the pull-down state and the contactless one, where charging is induced under the presence of much lower electric field intensities with the moving armature being still in the pull-up state [10]. The later has been shown to have polarity opposite of the contacted one [10] thus giving rise to a compensation mechanism, but the effect has not been further investigated.

Taking these into account the present paper aims to provide a better knowledge of the characteristics of the two mechanisms, by presenting information on the characteristic time constants and charging/discharging current magnitudes. This in turn will allow the understanding of the degree of compensation process, hence the mitigation of dielectric charging by proper engineering of the dielectric material.

\section{Theoretical background}

\subsection{Charging mechanisms}

In principle the charging/polarization of a dielectric material is achieved through two basic processes: (i) the contact-less, termed as blocking contacts polarization [11], where the electrodes generating the electric field are not in contact with the film, hence no charge is injected and (ii) the contacted one where the dielectric film is in contact with the electrodes generating the electric field allowing charge injection (Fig. 1). In the first case the charging 


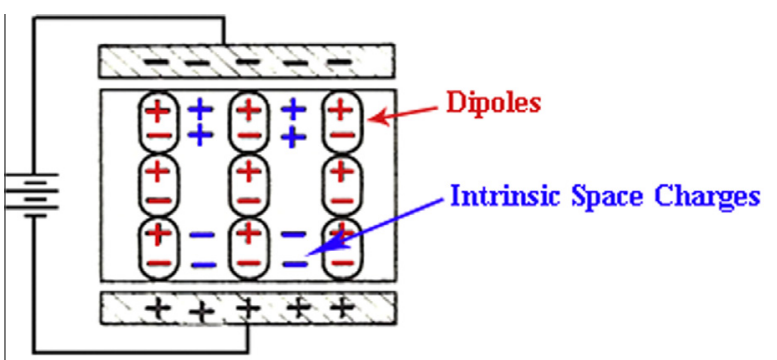

(a)

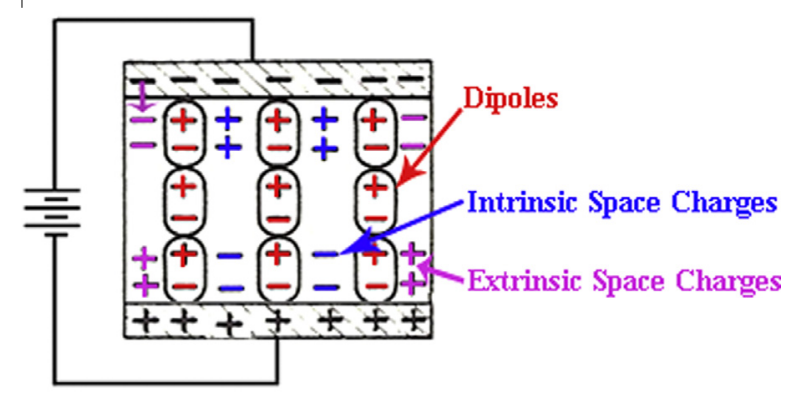

(b)

Fig. 1. (a) Contact-less and (b) contacted charging.

arises only from dipole orientation, which generates a charge density $\sigma_{\text {dip }}$ in the dielectric, and intrinsic free charge displacement, a mechanism that produces a charge density $\sigma_{\text {int }}$. In the second case the charge injection provides an additional mechanism to overall charging, with a corresponding charge density $\sigma_{\text {ext }}$. The two mechanisms, that are the dipole orientation and the intrinsic free charge displacement, give rise to same polarity charging and the contribution of each mechanism can be identified with methods based on DC and AC characterization.

In a MEMS capacitive switch the first mechanism, the contactless charging, is monitored under low electric field intensities because the moving armature has to be in the pull-up state [11] in order to avoid charge injection. Both mechanisms, contact-less and contacted charging, occur under the presence of the high electric field intensities during the pull-down state. In the specific case of capacitive switches with stiff bridge and therefore with large pull-down voltage, the case of power devices operating at bias levels close but below pull-down, field emission may occur. Then charges may be injected into dielectric film and give rise to "contacted" charging, thus producing an additional charge density $\sigma_{e x t}^{\prime}$ in the dielectric film, while the moving armature is still in the pull-up state $[12,13]$. Here it must be pointed out that this condition can be achieved by the superposition of power RF signal, the different work functions of the moving armature metal and the dielectric film composition. This phenomenon may give rise to charging current that will affect the shift rate of pull-down and pull-up voltages with the number of cycles and therefore it will affect the lifetime of the switches.

According to these, the total charge density $\sigma$ that arises in a dielectric film of a MEMS capacitive switch during its operation may be written as:

$\sigma=\left(\sigma_{\text {ext }}+\sigma_{\text {ext }}^{\prime}\right)-\left(\sigma_{\text {dip }}+\sigma_{\text {int }}\right)$

where $\sigma_{\text {dip }}$ arises from dipole orientation, $\sigma_{\text {int }}$ from intrinsic space charges, $\sigma_{\text {ext }}$ from extrinsic space charges due to metal-dielectric contact and $\sigma_{\text {ext }}^{\prime}$ comes from extrinsic space charges that are injected in the dielectric film due to field emission, as already mentioned above.

\subsection{Kelvin probe method in MEMS}

The bulk discharge current transient in a MEMS capacitive switch is determined using the device model proposed in $[14,15]$, which includes a fixed non-flat metal plate of area $A$ covered with a dielectric film of uniform thickness $d_{\varepsilon}$ and dielectric constant $\varepsilon_{r}$. In the general case of distributed equivalent charge and air gap [16], the bias at which the up-state capacitance attains its minimum $\left(V_{\min }\right)$ is the one for which the electrostatic force attains minimum. For small bridge up-state deformation the capacitance variance can be considered low and the bias for capacitance minimum is given by:

$V_{\min }(t)=\frac{d_{\varepsilon}}{\varepsilon_{r} \varepsilon_{0}} \cdot \mu_{\psi}+V_{\text {offset }}$

where $\mu_{\psi}$ represents the mean value of the equivalent surface charge distribution of the dielectric film and $V_{\text {offset }}$ arises from background charging of substrate [17], ESD, etc. The value of $V_{\min }$ will shift after each stress step, and this shift is determined by the stressing time or number of cycles and the applied electric field intensity. The value of $V_{\min }$ will also shift during the discharge process.

Taking all these into account the calculated net charging $\left(J_{c h}\right)$ or discharging $\left(J_{\text {disch }}\right)$ current density will be given by:

$J_{\text {ch,disch }}(t)=\frac{d \mu_{\psi}}{d t}=\frac{\varepsilon_{r} \varepsilon_{0}}{d_{\varepsilon}} \cdot \frac{d V_{\min }(t)}{d t}$

which describes the average value of current density due to charge injection and storage or charge collection by the bottom electrode. Moreover, the stored or collected charge density, $\sigma_{c h}$ or $\sigma_{\text {disch }}$, can be calculated by integrating the charge/discharge current density within the time window of observation:

$\sigma_{c h, d i s c h}(t)=\int_{t_{0}}^{t} J_{c h, d i s c h}(t) \cdot d t$

\section{Experimental procedure}

The switches measured are bridge-type capacitive switches with $70 \mathrm{~nm}$ thick $\mathrm{SiN}_{x}$ dielectric film, deposited with PECVD method at $350{ }^{\circ} \mathrm{C}$. The membrane is suspended about $2 \mu \mathrm{m}$ above the dielectric in the unactuated position and the active area is about $1.3 \times 10^{-4} \mathrm{~cm}^{2}$. The devices used in the present work were selected for low pull-down voltage, $V_{p d} \cong 13 \mathrm{~V}$, in order to avoid excess charging during contacted stress. For the contacted charging the devices were stressed for a total time of $1020 \mathrm{~s}$ in steps of $30 \mathrm{~s}$ at $15 \mathrm{~V}$. The contactless charging was performed under bias of $+8 \mathrm{~V}$ and for a total time of $7200 \mathrm{~s}$ in steps of $900 \mathrm{~s}$. After stress the devices were assessed by obtaining the $\mathrm{C}-\mathrm{V}$ characteristics in the up state for $5500 \mathrm{~s}$ in order to monitor the shift of the bias for minimum capacitance towards the pre-stress level.

The charging and discharging processes have been monitored through the bias for up-state capacitance minimum with the aid of a Boonton $72 \mathrm{~B}$ capacitance meter while sweeping the voltage in $50 \mathrm{mV}$ steps and the acquired bias was applied to the bottom electrode by a 6487 Keithley voltage source-picoampere meter. Here it must be pointed out that the measured capacitance included the parasitic one induced by the low frequency probes. The minimum of the $\mathrm{C}-\mathrm{V}$ characteristics was determined by fitting a parabola to the experimental data, assuming a small capacitance variance in order to derive Eqs. (2) and (3), in agreement to the theory proposed in $[14,16]$. Finally, all measurements have been performed under vacuum, with prior annealing of the samples at a temperature of $140{ }^{\circ} \mathrm{C}$ for $2 \mathrm{~h}$, in order to avoid any interference from humidity. 


\section{Results and discussion}

The shift of the $\mathrm{C}-\mathrm{V}$ characteristic with stress time for contactless charging is shown in Fig. 2. The contactless charging was performed under a bias of $+8 \mathrm{~V}$, that corresponds to an applied electric field of the order of $4 \times 10^{4} \mathrm{~V} / \mathrm{cm}$, resulting in a shift to more negative voltages. For contacted charging the devices have been stressed under bias of $+15 \mathrm{~V}$, that corresponds to an electric field of $2.1 \times 10^{6} \mathrm{~V} / \mathrm{cm}$.

The shift of the bias for minimum capacitance $\left(V_{\min }\right)$ for the case of contactless and contacted charging is shown in Fig. 3a and b respectively. Due to the large difference of stressing electric fields the shift is larger and faster for contacted charging with respect to the shift for contactless charging. It is important to emphasize that during contactless charging the dipole orientation and charge translation will decrease the internal electric field intensity that will in turn decrease the dipoles alignment and free charge translation rate with time (Fig. 3a). On the other hand in the case of contacted charging the electric field at the dielectric-metal contact decreases with charging time hence decreasing the rate of charge build-up in the dielectric (Fig. 3b). These charging processes include the effect of charge trapping, hopping [18] and percolation and they will lead to a macroscopic behavior that obeys the stretched exponential law $[14,15]$ with time constants of $48 \mathrm{~s}$ and $1005 \mathrm{~s}$ for the contacted and contactless charging respectively. The low value of charging time can be attributed to the large applied electric field and mainly to the short observation time window. Moreover the stretched exponential factor $\beta$ has been found to be smaller for the case of contacted charging, as presented in Fig. 3, thus revealing that this process is probably more complex than the one referring to contactless charging.

The charging current for each process has been also calculated according to Eq. (3) and it has been found to decrease exponentially with time, with corresponding time constants of $104 \mathrm{~s}$ and $4425 \mathrm{~s}$ for the contacted and contactless charging respectively (Fig. 4). The total equivalent charge density has been calculated from Eq. (4) and leads to $8.85 \times 10^{-8} \mathrm{C} / \mathrm{cm}^{2}$ for the contacted and $2.45 \times 10^{-8} \mathrm{C} / \mathrm{cm}^{2}$ for the contactless charging. It is interesting to notice that the equivalent charge for the contactless charging, although accumulated in a long time $(\sim 7200 \mathrm{~s})$, is non-negligible compared to the one from contacted charging. Despite the fact that contacted charging is expected to accumulate large amount of charge in a long term, the calculated value for contactless charging raises the question of whether an effective compensation would result in a longer lifetime. This can only be achieved if the contactless charging will arise from dipole orientation and/or ion

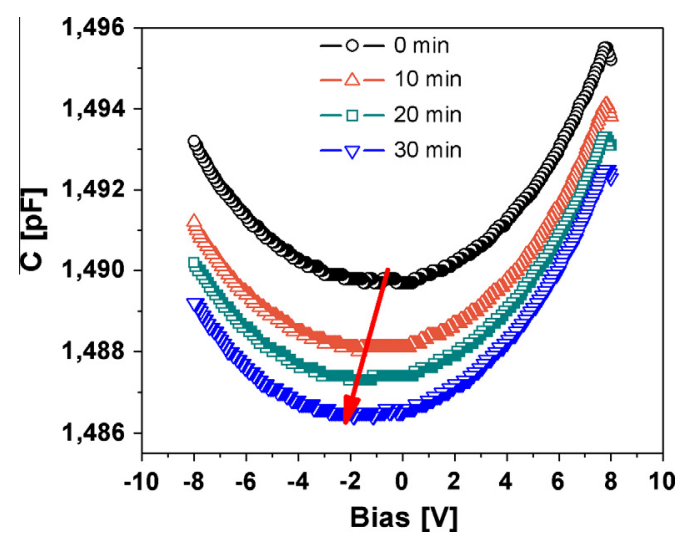

Fig. 2. Shift of capacitance-voltage characteristics after successive contactless charging.
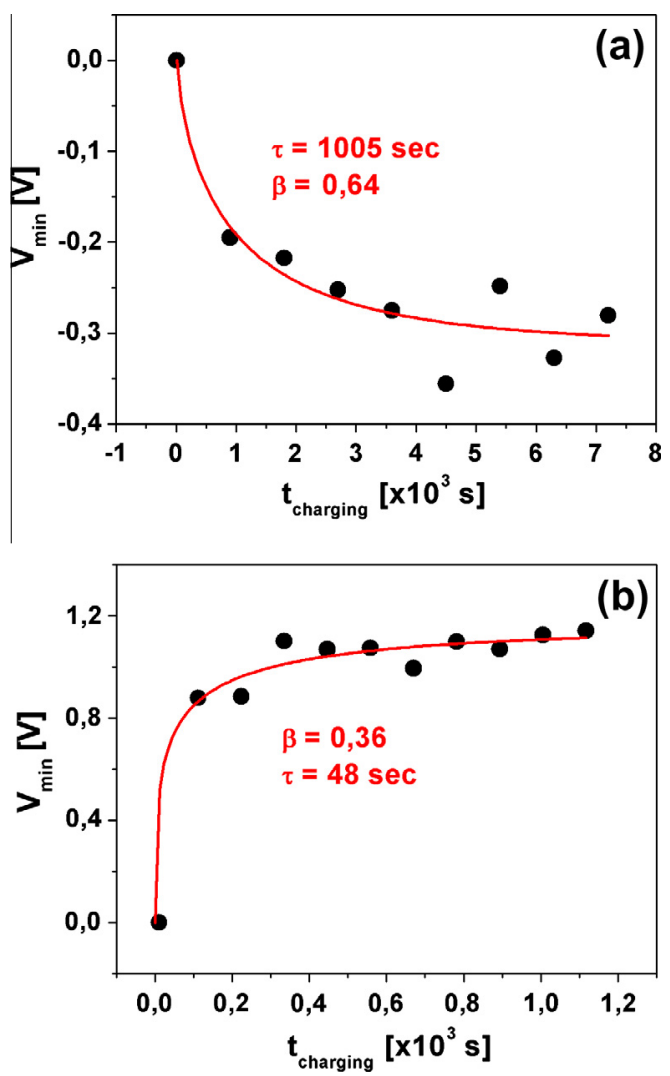

Fig. 3. Shift of the bias for capacitance minimum for (a) contactless and (b) contacted charging.

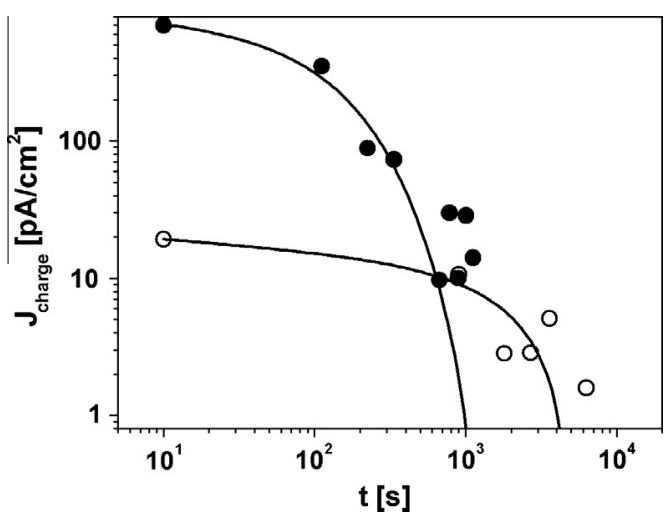

Fig. 4. Charging current densities for $(0)$ contactless and $(\bullet)$ contacted charging.

translation, since the charging from free charge translation will be immediately annealed.

The discharge process after contacted and contactless charging has been also investigated and correlated. Fig. 5a presents the shift of the bias for minimum up-state capacitance after contactless charging. The time constant of this process has been found to be equal to $1097 \mathrm{~s}$, a value very close to the characteristic time of the contactless charging process. Moreover there is a small potential offset in $V_{\min }$ of about $-0.20 \mathrm{eV}$ as shown in Fig. 5a. This offset arises during the time window of observation due to dipoles that have not been reoriented and/or ions that are collected under very long time constants. As far as it concerns the discharging process after contacted charging, the time constant is found to be $980 \mathrm{~s}$ and thus of the same order as the corresponding time after contactless charging, as shown in Fig. 5b. 

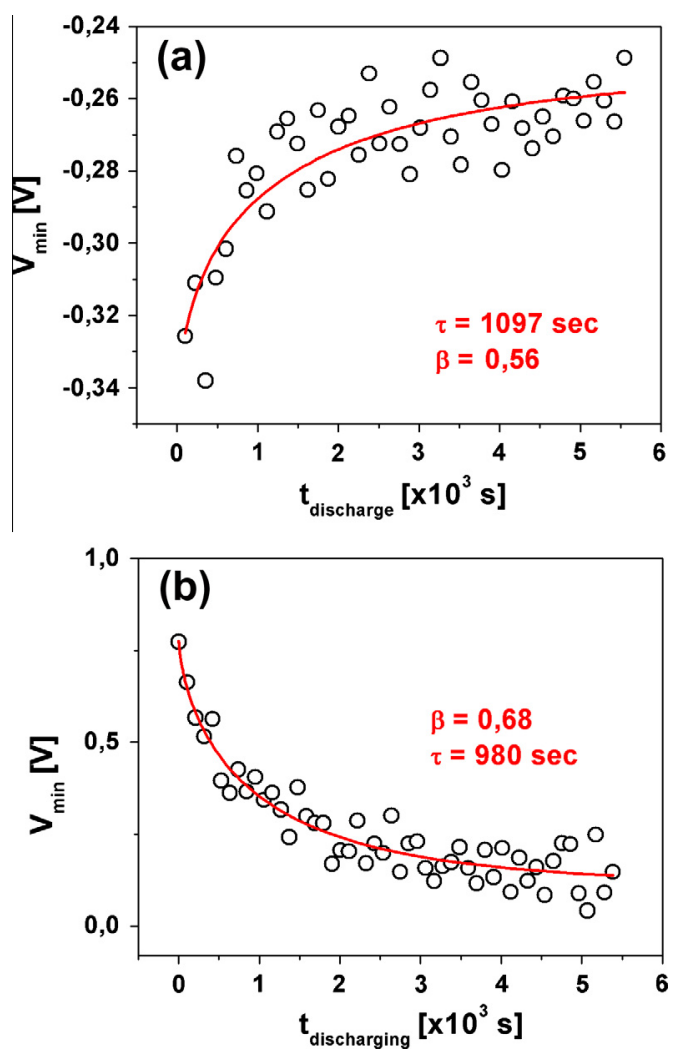

Fig. 5. Shift of the bias for minimum capacitance during discharge, after (a) contactless and (b) contacted charging.

According to these, the discharge process is found to be similar for both cases, i.e. after contactless and contacted charging, since the charge decay obeys a stretched exponential law during discharge, with similar relaxation time $(\tau)$ and stretched exponential factor $(\beta)$ (Fig. 5). The discharge mechanism depends on the dielectric material properties and so in both cases the dominant conduction mechanism during discharge is expected to be variable range hopping [18].

\section{Conclusions}

The present paper investigates and correlates the properties of contacted and contactless charging that occurs in the dielectric films of MEMS capacitive switches. Charge accumulation follows stretched exponential law for both contacted and contactless charging processes. The characteristic time of contacted charging has been found to be much smaller than the corresponding time of contactless charging and the magnitude of the charging current is much larger for the case of contacted charging. Despite these, the equivalent charge density for the two processes has been found to be similar in magnitude. Finally, the discharge process that follows contactless charging has the same characteristics with the one that follows contacted charging, since the charge collection mechanism depends on the properties of the dielectric material and both take place under relatively low electric fields.

In order to obtain a better understanding of charging and discharging processes in dielectric films of MEMS capacitive switches it is essential to bear in mind that during charging the electric field is high so that the hopping effect can be considered negligible leaving the Poole-Frenkel effect to control the charge redistribution.
But after pull-out, the discharge is performed under low electric field where variable range hopping dominates this process $[18,19]$.

The obtained results of this paper raise the question of whether an effective compensation due to contactless charging would be exploited resulting in a slower charging rate and therefore a longer lifetime of a MEMS capacitive switch. This constitutes an issue that presently is under further investigation.

\section{Acknowledgements}

The present work has been supported by FP7 project: "Nanostructured materials and RF-MEMS RFIC/MMIC technologies for highly adaptive and reliable RF systems", NANOTEC Grant Agreement No.: 288531. M. Koutsoureli acknowledges the support of the Operational Program "Education and Lifelong Learning" of the National Strategic Reference Framework (NSRF)-Research Funding Program: "Heracleitus II Investing in knowledge society through the European Social Fund.

\section{References}

[1] Yao JJ. RF MEMS from a device perspective. J Micromech Microeng 2000;10:R9-R38.

[2] Rebeiz GM. RF MEMS theory, design, and technology. Hoboken, NJ: Wiley; 2003.

[3] Wibbeler J, Pfeifer G, Hietschold M. Parasitic charging of dielectric surfaces in capacitive microelectromechanical systems (MEMS). Sens Actuators, A 1998;71:74-80.

[4] Goldsmith C, Ehmke J, Malczewski A, Pillans B, Eshelman S, Yao Z, et al. Lifetime Characterization of Capacitive RF MEMS Switches. In: IEEE international microwave symposium. vol. 1; 2001. p. 227-30.

[5] Czarnecki P, Rottenberg X, Puers R, De Wolf I. Effect of gas pressure on the lifetime of capacitive RF MEMS switches. In: 19th IEEE international conference on micro electro mechanical systems, MEMS; 2006. p. 890-3.

[6] Zaghloul U, Bhushan B, Pons P, Papaioannou GJ, Coccetti F, Plana R. On the influence of environment gases, relative humidity and gas purification on dielectric charging/discharging processes in electrostatically driven MEMS/ NEMS devices. Nanotechnology 2011;22:035705.

[7] Ruan J, Papandreou E, Lamhamdi M, Koutsoureli M, Coccetti F, Pons P, et al. Alpha particle radiation effects in RF MEMS capacitive switches. Microelectron. Reliab 2008;48:1241-4.

[8] Papaioannou G. Advanced RF MEMS. In: Lucyszyn S, editor. The cambridge RF and microwave engineering series; 2010. [Chapter 6]

[9] Van Spengen WM. Capacitive RF MEMS switch dielectric charging and reliability: a critical review with recommendations. J Micromech Microeng 2012;22:074001.

[10] Papaioannou G, Wang G, Bessas D, Papapolymerou J. Contactless dielectric charging mechanisms. In: RF-MEMS capacitive switches, European microwave week; 11-13 September. 2006. p. 513-6.

[11] Vandershueren J, Casiot J. Topics in applied physics. In: Braunlich P, editor. Thermally stimulated relaxation in solids, vol. 37. Berlin: Springer-Verlag; 1979. p. 135 [Chapter 4].

[12] Garg A, Venkattraman A, Kovacs A, Alexeenko A, Peroulis D. Direct measurements of field emission currents in e-static MEMS structures. In: IEEE MEMS conference; 2011. p. 412-5.

[13] Michalas L, Garg A, Venkattraman A, Koutsoureli M, Alexeenko A, Peroulis D, et al. A study of field emission process in electrostatically actuated MEMS switches. Microelectron Reliab 2012;52:2267-71.

[14] Koutsoureli M, Papaioannou G. Determination of long time discharge current in microelectromechanical system capacitive switches. Appl Phys Lett 2011;99:103503.

[15] Koutsoureli M, Michalas L, Papaioannou G. Temperature effects on the bulk discharge current of dielectric films of MEMS. Microelectron Reliab 2012;52:2240-4.

[16] Rottenberg X, De Wolf I, Nauwelaers BKJC, De Raedt W, Tilmans HAC. Analytical model of the dc actuation of electrostatic MEMS devices with distributed dielectric charging and nonplanar electrodes. J Microelectromech Syst 2007;16:1243-53.

[17] Czarnecki P, Rottenberg X, Soussan P, Ekkels P, Muller P, Nolmans P, et al Effect of substrate charging on the reliability of capacitive RF MEMS switches. Sens Actuators, A 2009;154:261-8.

[18] Koutsoureli M, Michalas L, Papaioannou G. Charge collection mechanisms in MEMS capacitive switches. In: IEEE international reliability physics symposium (IRPS), Anaheim, CA, USA; 2012. p. M.E.2.1-.5.

[19] Koutsoureli M, Michalas L, Papaioannou G. The effect of temperature on dielectric charging of capacitive MEMS. In: IEEE international reliability physics symposium (IRPS), Monterey, CA, USA; 2011. p. 3D.4.1-.7. 